\title{
Research Paper: Functional Safety Status in Isfahan Private Hospitals in Response to Disasters in 2015
}

\author{
Nahid Tavakoli $^{1^{*}}$, Mahdieh Mousavi
}

1. Health Management and Economics Research Center, Isfahan University of Medical Sciences, Isfahan, Iran.

2. Department of Management and Health Information Technology, Faculty of Management and Medical Information, Isfahan University of Medical

Sciences, Isfahan, Iran.

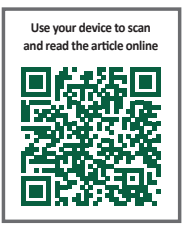

Citation: Tavakoli N, Mousavi M. Functional Safety Status in Isfahan Private Hospitals in Response to Disasters in 2015. Health in Emergencies and Disasters Quarterly. 2017; 2(3): 125-132. https://doi.org/10.18869/nrip.hdq.2.3.125

Article info:

Received: 09 Dec. 2016

Accepted: 23 Feb. 2017

\section{Keywords:}

Assessment, Patient safety, Hospital safety, Functional safety, Private hospital

\section{ABSTRACT}

Background: Hospital safety is of great social importance in various aspects, including functional safety. If hospitals fail to response appropriately to a disaster or protect people against the risks resulting from natural and man-made disasters, people feel insecure and society loses the stability. The present article aims to evaluate the functional safety status of hospitals in disasters.

Materials and Methods: This research is a descriptive cross-sectional study conducted in 2015. The study population consisted of all private hospitals in Isfahan and the sampling method was census. In this study, WHO Hospital Safety Index checklist, which is used for vulnerability assessment, was used as the research tool and data analysis was done by Excel.

Results: According to the results, the mean functional safety of Isfahan private hospitals were 1.63 for "organizing hospital emergency/disaster committee," 1.2 for "action plan to respond to internal and external risks," 0.5 for "contingency plans of medical operations," 1.62 for "access to operational plans of preserving and restoring vital services," and 0.8 for "access to medicines, equipment and supplies needed in emergency situations." Overall, the functional safety of hospitals was moderate.

Conclusion: The present study showed that the current status of functional safety in Isfahan private hospitals is not completely satisfactory and the provincial health managers, especially Isfahan University of Medical Sciences, are expected to regularly monitor planning and decision-making about the risks threatening these hospitals, especially in the areas of contingency plans of medical operations and access to medicines as well as equipment and supplies needed in an emergency situation.

\section{Introduction}

afe hospitals maintain their operational and functional capacity and capability during and even after the emergency situ- ation and can continue their operation appropriately in terms of structural, nonstructural, and functional constructs during and after the emergency. Thus improving their safety is of utmost importance [1]. Hospitals

* Corresponding Author:

Nahid Tavakoli, PhD

Address: Health Management and Economics Research Center, Isfahan University of Medical Sciences, Isfahan, Iran.

E-mail:tavakoli@mng.mui.ac.ir 
are expected to play a key role in reducing injuries and fatalities [2]. Also, their preparedness to respond effectively in critical situations and minimize complications and loses arising from disasters is undeniable [3].

The World Health Organization has promoted some plans for safer hospitals at local and national levels to strengthen hospitals in response to emergency situations. As well, in order to help hospital managers for assessing structural and functional safety of hospitals and improving their readiness to respond to emergencies, WHO has prepared checklists to assess the capacity of the health system in a crisis situation [4]. Also, a lot of research has been conducted in recent decades on patient safety improvement as well as the risks, causes, effects, and the methods used to improve safety and reduce the risks and adverse effects of disasters [3] which indicate the importance of assessment and determination of accurate and transparent tasks, responsibilities and authorities of all individuals and organizations involved in disaster management, especially hospitals, to protect people against natural and man-made threats [5].

Hospital safety culture is one of the main sectors that provide high-quality services and promote secure environment for the safety of patients and staff, particularly in critical conditions. Thus, it is necessary to identify potential capabilities and safety status of hospitals for involving them in response to emergencies [6]. Watson considered hospital safety as a crisis and believed that it is time to propose this important issue in the community and thereby, all responsible public health centers should provide important services to improve hospital safety and promote patient safety [7].

Some studies in Iran suggest that the weak construction of some medical centers together with the vulnerability of communication network and non-compliance with design criteria can cause dysfunction and inefficiency of medical centers during the disaster [8,9]. Habibi assessed the general safety status of the radiology wards of Isfahan University hospitals as moderate and poor; in which, the highest risk percentage belonged to the moderate risks and the lowest percentage to the very important risks [10]. Valcárcel assessed structural and non-structural safety of Catholic hospitals in Spain and stressed that hospital vulnerability assessment is necessary in the area of functional safety [8]. The results of Pisla study (2010), who assessed the safety of 66 hospitals in the Maldives, showed that about $25 \%$ of hospitals are highly resilient against sudden disaster, about $25 \%$ are moderately resilient and the rest are not resilient. Also, the weighted mean of the indexes showed that the hospitals had the lowest functional safety [11]. Of the most important activities in the field of healthcare is the risk analysis and assessment of disaster risk in all structural, nonstructural, and functional aspects of hospitals and health centers and reduction of their liability through identifying and assessing the vulnerabilities and their correction $[2,12]$.

In this regard, hospitals play a key role in disaster management and take important responsibilities, including diagnosis and treatment services to the victims of disasters and minimizing the fatalities and casualties of disasters, admission and accommodation of patients discharged from hospitals or other centers, formation and dispatch of emergency medical teams, triage of the victims during massive disasters, providing health and mental health services to patients and society as well as public awareness. Thus it is necessary to assess the hospitals in terms of readiness against emergency situations with regard to safety, including functional safety.

By assessing the hospital safety index and calculating the safety level against a disaster, we can determine to what extent a hospital can maintain its structure and function during a disaster. Using these indicators and information, decision- and policy-makers would be able to prioritize management and construction interventions in the healthcare area $[13,14]$. Several studies have been conducted in the field of patient and hospital safety, but researchers have mostly focused on patient safety, structural safety, and non-structural safety of hospitals [15 -17], however functional safety as an important aspect of hospital safety index has got less attention. This study aims to assess the functional safety status in Isfahan private hospitals in response to disasters and provide strategies to increase preparedness against emergencies and also improve safety in hospitals.

\section{Materials and Methods}

This research is a descriptive cross-sectional study conducted in fall and winter 2015. The study population comprised all private hospitals in Isfahan and the sampling method was census. For data collection, WHO Hospital Safety Index checklist was used. It is targeted to assess structural and functional safety of hospitals and there is no need to verify its validity and reliability. But for the purpose of this study, only the questions related to functional safety in the checklist, i.e. 61 items were used. The checklist consists of two parts: first part includes general information of hospital and the second part contains five fields related to functional safety, including "organizing hospital emergency/disaster com- 
mittee," "action plan to respond to internal and external risks," "contingency plans of medical operations," "access to operational plans of preserving and restoring vital services," and "access to medicines, equipment and supplies needed in emergency situations". We denote 0 to answer the questions with low score, 1 for average score and 2 for high score.

The researcher visited all private hospitals in Isfahan (6 hospitals) and coordinated with the hospital disasters and emergencies management and also observed the current status of the hospital, Then, completed the checklist items in different wards during several visits. The information was imported to Excel and the numerical values were derived from it. According to the total scores of 122 (from the questionnaire), the results of functional safety with a score of 90-122 were considered great, 60-90 good, 30-60 average, and below 30 poor.

\section{Results}

The results of the data analysis on 6 questionnaires, including 61 items and 5 areas showed that the functional safety of Isfahan private hospitals is in the average level. In this section, the results are presented with respect to the research objectives.

With regard to the first objective, findings showed that the average score of hospitals in organizing hospital emergency/disaster committee was 1.4. In this area, the highest score belonged to the selection of committee members from different disciplines (1.83) and the lowest score was related to the protection and safety of hospital emergency operations command center location [1] (Table 1). According to the indicators of this field, hospital $E$ with a total score of 22 and the average score of 2 has the highest level in the field of "organizing hospital emergency/disaster committee".

The score of hospitals in the area of "action plan to respond to internal and external risks" was 0.78 . The highest score was related to "access to the emergency exit doors and other open exit routes" with 1.7 and the lowest score was related to "hospital epidemiological surveillance system" with 0.2. Hospitals C and F gained the highest score for the indicators of this area and have

Table 1. Mean functional safety in the field of organizing hospital emergency/disaster committee in Isfahan private hospitals

\begin{tabular}{|c|c|c|c|c|c|c|c|c|}
\hline $\begin{array}{l}\text { Organizing Hospital } \\
\text { Disaster Committee }\end{array}$ & A & B & C & D & $\mathbf{E}$ & $\mathbf{F}$ & Total & Mean \\
\hline $\begin{array}{l}\text { Forming an official committee to respond to emergencies, } \\
\text { disasters, or crisis }\end{array}$ & 1 & 2 & 1 & 2 & 2 & 2 & 10 & 1.6 \\
\hline Selecting committee members from different disciplines & 1 & 2 & 2 & 2 & 2 & 2 & 11 & 1.83 \\
\hline Awareness of each member about their responsibilities & 0 & 1 & 2 & 2 & 2 & 2 & 9 & 1.5 \\
\hline $\begin{array}{l}\text { Determining a location for hospital Emergency Operations } \\
\text { Center (EOC) }\end{array}$ & 1 & 1 & 0 & 1 & 2 & 2 & 7 & 1.16 \\
\hline Protection and safety of the EOC location & 0 & 2 & 0 & 2 & 2 & 0 & 6 & 1 \\
\hline Internet network and computer equipment of the EOC & 1 & 2 & 2 & 1 & 2 & 2 & 9 & 1.5 \\
\hline $\begin{array}{c}\text { The operation of internal and external communication } \\
\text { systems of the EOC }\end{array}$ & 1 & 1 & 2 & 1 & 2 & 2 & 9 & 1.5 \\
\hline An alternative communication system for the EOC & 0 & 1 & 1 & 1 & 2 & 2 & 7 & 1.16 \\
\hline Sufficient equipment in the EOC & 1 & 1 & 2 & 2 & 2 & 1 & 9 & 1.5 \\
\hline Updated phone book in the EOC & 1 & 0 & 2 & 2 & 2 & 1 & 8 & 1.16 \\
\hline Job description for all personnel & 1 & 0 & 2 & 1 & 2 & 2 & 8 & 1.3 \\
\hline Total scores of organizing emergency/disaster committee & 7 & 13 & 16 & 17 & 22 & 18 & 103 & 83 \\
\hline $\begin{array}{l}\text { The mean score of organizing emergency/disaster com- } \\
\text { mittee }\end{array}$ & 0.63 & 1.18 & 1.45 & 1.54 & 2 & 1.63 & 9.36 & 1.39 \\
\hline
\end{tabular}


Table 2. The mean functional safety in the area of contingency plans of medical operations in Isfahan private hospitals

\begin{tabular}{|c|c|c|c|c|c|c|c|c|}
\hline $\begin{array}{l}\text { Contingency Pans of } \\
\text { Medical Operations }\end{array}$ & A & B & C & D & $\mathbf{E}$ & $\mathbf{F}$ & Total & Mean \\
\hline Earthquake or tsunami or volcano or landslide & 0 & 1 & 0 & 0 & 0 & - & 1 & 0.16 \\
\hline Social conflicts and terrorism & 0 & 0 & 0 & 0 & 0 & 0 & 0 & 0 \\
\hline Flood and storm & 0 & 0 & 0 & 0 & 0 & - & 0 & 0 \\
\hline Fire or explosion & 0 & 2 & 1 & 0 & 2 & 1 & 6 & 1 \\
\hline Chemical accidents or exposure to ionizing radiation & 0 & 0 & 0 & 0 & 0 & 0 & 0 & 0 \\
\hline Epidemics & 0 & 0 & 1 & 0 & 1 & 1 & 3 & 0.5 \\
\hline $\begin{array}{l}\text { Treatment and psychological and social support for patients, families } \\
\text { and staff }\end{array}$ & 0 & 0 & 0 & 0 & 0 & 0 & 0 & 0 \\
\hline Hospital-acquired infections control & 1 & 0 & 1 & 0 & 1 & 2 & 5 & 0.83 \\
\hline Contingency plans of medical operations & 1 & 3 & 3 & 0 & 4 & 4 & 15 & 2.49 \\
\hline Mean score of contingency plans of medical operations & 0.12 & 0.37 & 0.37 & 0 & 0.5 & 0.5 & - & 0.31 \\
\hline
\end{tabular}

more comprehensive action plan than other hospitals in response to internal and external risks. The results of mean functional safety in the area of "contingency plans of medical operations" are provided in Table 2.
On the whole, the hospitals failed to get good scores in this area and evaluated at weak level. In this area, the indicator of "fire and explosion" with an average of 1 had the highest score than the other indicators. Analysis of the results in the field of "access to operational plans

Table 3. The mean functional safety in the area of access to operational plans of preserving and restoring vital services in Isfahan private hospitals

\begin{tabular}{|c|c|c|c|c|c|c|c|c|}
\hline $\begin{array}{l}\text { Access to Operational Plans } \\
\text { of Preserving and Restoring Vital Services }\end{array}$ & A & B & C & D & E & $\mathbf{F}$ & Total & Mean \\
\hline Auxiliary power sources and generators & 0 & 2 & 1 & 0 & 2 & 2 & 7 & 1.16 \\
\hline Reserves of drinking water & 0 & 0 & 2 & 1 & 2 & 2 & 7 & 1.16 \\
\hline Reserves of fuel & 0 & 1 & 2 & 0 & 1 & - & 4 & 0.66 \\
\hline Medical gases & 0 & 1 & 2 & 0 & 2 & 2 & 7 & 1.16 \\
\hline Communication and backup systems & 0 & 0 & 2 & 1 & 2 & 2 & 7 & 1.16 \\
\hline Sewage systems & 2 & 1 & 2 & 0 & 2 & 2 & 9 & 1.5 \\
\hline Solid waste management & 1 & 2 & 2 & 0 & 0 & 2 & 7 & 1.16 \\
\hline Repair and maintenance of fire protection systems & 1 & 2 & 1 & 1 & 2 & 1 & 8 & 1.33 \\
\hline $\begin{array}{l}\text { Accessibility of operational plans of preserving and restor- } \\
\text { ing vital services }\end{array}$ & 4 & 8 & 14 & 3 & 13 & 13 & 56 & 9.29 \\
\hline $\begin{array}{c}\text { Mean score of access to operational plans of preserving } \\
\text { and restoring vital services }\end{array}$ & 0.5 & 1 & 1.75 & 0.37 & 1.62 & 1.62 & - & 1.16 \\
\hline
\end{tabular}


Table 4. The mean functional safety within 5 areas in Isfahan private hospitals

\begin{tabular}{|c|c|c|c|c|c|c|c|}
\hline Hospital & A & B & $\mathbf{C}$ & D & $\mathbf{E}$ & $\mathbf{F}$ & $\begin{array}{l}\text { Total } \\
\text { Mean }\end{array}$ \\
\hline Organizing hospital emergency/disaster committee & 0.63 & 1.18 & 1.45 & 1.45 & 2 & 1.63 & 1.40 \\
\hline Action plan to respond to internal and external risks & 0.45 & 0.87 & 1.20 & 0.33 & 0.66 & 1.20 & 0.78 \\
\hline Contingency plans of medical operations & 0.12 & 0.37 & 0.37 & 0 & 0.5 & 0.5 & 0.31 \\
\hline $\begin{array}{l}\text { Access to operational plans of preserving and restoring vital } \\
\text { services }\end{array}$ & 0.5 & 1 & 1.75 & 0.37 & 1.62 & 1.62 & 1.14 \\
\hline $\begin{array}{l}\text { Access to medicines, equipment, and supplies needed in emer- } \\
\text { gency situations }\end{array}$ & 1.1 & 1.3 & 1.4 & 0.7 & 0.7 & 0.8 & 1 \\
\hline
\end{tabular}

of preserving and restoring vital services" in Isfahan private hospitals presented in Table 3 shows that hospitals $\mathrm{C}$ and $\mathrm{F}$ have the highest access to operational plans of preserving and restoring vital services. In this area, the indicators of "sewage systems" with an average of 1.5 and "repair and maintenance of fore protection systems" with an average of 1.33 had higher scores than the other indicators. The indicator "reserves of fuel" with an average of 0.66 achieved the lowest score in this area.

Finally, the analysis of results in the field of "access to medicines, equipment, and supplies needed in emergency situations" in Isfahan private hospitals showed that the hospitals had higher safety in this area. Hospitals $\mathrm{C}$ and $\mathrm{F}$ achieved the highest scores of the indicators related to this area and had more comprehensive action plan than the other hospitals in response to internal and external risks. Hospital $\mathrm{C}$ with a total score of 14 and an average of $1.4 \mathrm{had}$ the highest readiness in the area of "access to medicines, equipment and supplies needed in emergency situations" among other private hospitals. As well, the indicators of "medicine" and "medical gases" with an average of 1.5 gained the highest scores in this area. The overall results of the mean functional safety of hospitals in Isfahan private hospitals are provided in Table 4.

\section{Discussion}

This study aimed to determine functional safety of Isfahan private hospitals in 5 areas. The results showed that in the area of "organizing hospital emergency/disaster committee," hospital E with a total score of 22 was in desired status and hospital A with a total score of 7 was in poor status. The mean functional safety in the area of "action plan to respond to internal and external risks" in
Isfahan private hospitals indicated that hospitals $\mathrm{C}$ and $\mathrm{F}$ with a score of 29 gained the highest score in this field and had more comprehensive action plan than the other hospitals. These hospitals had obtained the highest score in the indictors of "transportation and support service status" and "regular safety inspections of hospital premises and equipment" and also the lowest score in the indicator of "necessary measures to ensure the welfare and health of auxiliary and volunteer personnel in emergency".

The mean functional safety in the area of contingency plans of medical operations indicated that hospital $\mathrm{E}$ has the highest score. In general, the hospitals obtained good scores in this area. Meantime, the indicator of "fire and explosion" had higher score than the other indices. The mean functional safety in the area of access to operational plans of preserving and restoring vital services in Isfahan private hospitals showed that hospitals $\mathrm{C}$ and $\mathrm{F}$ have the highest access to operational plans of preserving and restoring vital services while hospital D has gained the lowest score. Generally, all hospitals obtained higher scores in the indexes of "sewage system" and "repair and maintenance of fire protection systems" than the other indexes in this field.

The index of "fuel reserves" also achieved the lowest score. Finally, the findings of the mean functional safety in the area of "access to medicines, equipment and supplies needed in emergency situations" in Isfahan private hospitals showed that hospital $\mathrm{C}$ had higher safety in this area while hospital F was weak. Also, the indexes of "medicine" and "medical supplies" had gained the highest scores in this area. Regarding the applied objective of this study, assessing the vulnerabilities and readiness of private hospitals against disasters showed that hospital $\mathrm{C}$ obtained 
a score of 76 according to the standard indices and thus had more favorable safety status compared to other hospitals. The overall percentage of compliance with various safety indexes showed that hospital A with a score of 34 had the lowest score compared to other hospitals.

The present study showed that the functional safety status of Isfahan private hospitals was poor only in the area of contingency plans of medical operations. However the results of Khaloei et al. study at educational hospitals of Kerman University of Medical Sciences showed that the safety status was not desirable in most hospital wards and units [18]. Although Iran is a disaster-prone country, particularly vulnerable to earthquakes, floods, and chemical accidents, its hospitals are not prepared to deal with such disasters and no serious action has been taken so far.

Zhong in the study of resilience against disasters in 50 hospitals in China argued that assessing the risks that threaten the region as well as having an organized plan to discharge patients and vulnerable population of the region are among important approaches within the framework of hospital safety [19]. The results of Sabzeghabaei study on the status of safety and readiness in selected hospitals of Shahid Beheshti University of Medical Sciences and Mirzaee study in Ilam hospitals against disasters showed that the safety level is moderate in most hospitals $[20,21]$. However, the study of Seyedin on the assessment of educational hospitals of Tehran University of Medical Sciences reported the functional safety level desirable [22].

The study results were consistent with the above studies in some ways and inconsistent in some others, for example, in terms of "organizing hospital emergency/ disaster committee and access to operational plans of preserving and restoring vital services," the results were consistent with the results of Seyedin et al. study and inconsistent with other areas, particularly "contingency plans of medical operation". The study results of Pisla et al. (2010), entitled "Safety assessment in 66 hospitals in the Republic of Maldives", showed that about 25\% of the hospitals were in Group A with high resilience against disasters, about $25 \%$ of the hospitals in Group B with moderate resilience, and the rest were in Group C with no resilience. In addition, the weighted mean of the indexes showed that the hospitals had the lowest level of functional safety. Promoting the hospitals administration and holding training program were the recommendations of this study [11].

A part of the present research results, including contingency plans of medical operation, were consistent with the study. Hook study in the field of hospital safety culture in 6000 hospitals in the United States indicated that organizational learning and continuous improvement of processes are noticeable and significant to maintain the hospital safety culture [6]. Asefzadeh study on the risk assessment in Qazvin hospitals according to the World Health Organization model showed that the functional safety level is moderate in the hospitals [23]. Another part of the present research results, including action plan to respond to internal and external risks, were consistent with Asefzadeh study findings, while the rest were inconsistent. The current situation can be improved by developing and defining standard and integrated concepts of needed functional safety in medical centers to deal with disasters; preparing compiled training packages to promote the awareness, knowledge, and performance of managers, staff, and medical board of hospitals in the field of preservation and improvement of the functional safety; and finally allocating a part of the hospital accreditation scores to the documentation related to functional safety.

The limitation of this study was limited literature on the functional safety of hospitals, despite our extensive search in electronic databases and print resources. Accordingly, the researcher was obliged to use partly relevant articles in drafting the final report and paper.

\section{Conclusion}

The study findings showed that the functional safety status in Isfahan private hospitals against disasters was at moderate level; in particular the hospitals had the best score in the area of "organizing hospital emergency/disaster committee" and the lowest score in the field of "contingency plans of medical operations". Therefore; establishing an official committee to respond to an emergency, disaster, or crisis; selecting the committee members from different disciplines; and also favoring development and equipping the hospital EOC with appropriate communication facilities appear to be among important components of functional safety of hospitals. However, the safety status in the area of "contingency plans of medical operations" was evaluated as weak.

With respect to the subareas such as chemical accidents, hospital infections, epidemics, social conflicts and social support for patients, families and staff which are notable in this area, it is necessary for the hospital directors and managers to benefit from the services of other hospital committees and thus develop strategies to improve safety in this area to an acceptable level, including training personnel on how to deal with chemical accidents, hospital infections, and other risks and also how 
to save their lives and patients. In addition, the safety status is not satisfactory in the area of "action plan to respond to internal and external risks".

It reflects that hospitals have not taken appropriate and effective measures in important processes such as accepting emergency patients, regular inspection of safety of hospital premises and equipment, expansion of important hospital services (such as water, sewage, electricity, vital gases, etc.), the triage process, stabilization and treatment of patients and performing exercises and maneuvers in this regard. However, this process help respond to emergencies and disasters, hospital management in an emergency, protecting the safety of patients, hospital staff, and hospital itself. In sum, the study findings warn the managers of Isfahan private hospitals to foster the safety infrastructures to promote and maintain the safety culture of patient and hospitals for advancing the quality of health services.

\section{Acknowledgements}

This paper is extracted from the research project (No. 294135) funded by Isfahan University of Medical Sciences.

\section{Conflict of Interest}

The authors declared no Conflicts of Interest.

\section{References}

[1] Ahmad M, Murli JS. The importance of hospital safety in Indian scenario. Journal of the Academy of Hospital Administration. 2012; 23-24(1-2):5-54

[2] Djalali AR. Preparedness and safe hospital: Medical response to disasters [PhD thesis]. Stockholm: Karolinska Institutet, 2012.

[3] Dückers M, Faber M, Cruijsberg J, Grol R, Schoonhoven L, Wensing M. Safety and risk management interventions in hospitals: A systematic review of the literature. Medical Care Research and Review. 2009; 66(6):90-119. doi: $10.1177 / 1077558709345870$

[4] Rockenschaub G, Harbou KV. Disaster resilient hospitals: An essential for all-hazards emergency preparedness. World Hospitals and Health Services. 2013; 49(4):28-30. PMID: 24683813

[5] Radovic V, Vitale K, Tchounwou PB. Health Facilities Safety in Natural Disasters: Experiences and Challenges from South East Europe. International Journal of Environmental Research and Public Health. 2012; 9(12):1677-86. doi: 10.3390/ ijerph9051677
[6] Hook JN, Boan D, Davis DE, Aten JD, Ruiz JM, Maryon T. Cultural humility and hospital safety culture. Journal of Clinical Psychology in Medical Settings. 2016; 23(4):402-9. doi: 10.1007/s10880-016-9471-x

[7] Watson GL. The hospital safety crisis. Society. 2016; 53(4):339-47. doi: 10.1007/s12115-016-0028-2

[8] Valcárcel J, Pujades G, Barbat A. Integrated evaluation of hospital safety: Implications on resilience and welfare of communities. Paper presented at: The $14^{\text {th }}$ European Conference on Earthquake Engineering. 2010 August 30; Ohrid, Republic of Macedonia.

[9] Jahangiri, K. [Principles of disaster management (Persian)] Tehran: Helal University of Applied Science and Technology; 2011.

[10] Habibi E, Soleymanei B, Nateghei R, Lotfirosbehani M, Yarmohammadian, Yarmohammadian H. [Risk management in radiology units of Isfahan University of Medical Sciences' hospitals (Persian)]. Health Information Management. 2007 4(1):133-141.

[11] Pisla M, Domente D, Chetraru L, Ostaficiuc R. Evaluation of Hospital Safety in The Republic of Molova. Geneva: Republican Center for Disaster Medicine; 2010

[12] Radovic V, Vitale K, Tchounwou PB. Health facilities safety in natural disasters: experiences and challenges from south east Europe. International Journal of Environmental Research and Public Health. 2012; 9(12):1677-86. doi: 10.3390/ ijerph9051677

[13] Lari A, Jahangiri K, Haji Nabi K. [Hospital safety index analysis in confronting disasters: A case study (Persian)]. Scientific Journal of Rescue \& Relief. 2013; 5(1):1-9.

[14] Disaster Management Center. Principles of disaster management [Akbari Z, Tavakoli N, Raeisi A, Shams A, Sahebzadeh M, Keyvanara M, et al. Persian trans]. Isfahan: Isfahan University of Medical Sciences; 2009.

[15] Mohammadi Yeganeh Sh, Asadi-Lari M, Seyedin SH, Maher A. Qualitative and quantitative performance of equipment and non-structural vulnerability in selected public hospitals in Tehran City during an earthquake. Scientific Journal of Rescue \& Relief. 2011; 3(1-2):1-9.

[16] Jahangiri, Katayoun; Kheradmand, Mahnaz. [Evaluation of schools structural seismic vulnerability and choosing of safe buildings for emergency shelter (a case study) (Persian)] Scientific Journal of Rescue \& Relief. 2014; 6(2):2-16.

[17] Dixit AM, Guragain R. Methodology for assessing seismic vulnerabilities of health facilities. Regional Health Forum. 2009; 13(1):11-20

[18] Khaloei, A, Mehdi Pour Raberi M, Nakhaei N. The status of safety in training hospitals of Kerman University of Medical Sciences in 2010. Journal of Health and Development. 2013; 2(3):192-202.

[19] Zhong S, Hou XY, Clark M, Zang YL, Wang L, Xu LZ, et al. Disaster resilience in tertiary hospitals: a cross-sectional survey in Shandong Province, China. BMC Health Services Research. 2014; 14:135. doi: 10.1186/1472-6963-14-135

[20] Ardalan A, Sabzghabaee A, Najafi A, Zonoobi V. [The safety status in selected hospitals of Shahid Beheshti University of Medical Sciences against disaster compared to the standards 
during the years 2010 to 2012 (Persian)]. Tehran: Movaffagh Pub; 2010.

[21] Mirzaee F, Kakaei H, Farasati F, Zamani N. [Investigation on the safety status and preparedness of Ilam's hospitals against disasters in 2012 (Persian)]. Journal of Ilam University of Medical Sciences. 2015; 22(7):14-23.

[22] Seyedin H, Abasi Dolat Abadi Z, Sorani M, Naghdi S, Rajabfard Mazraeno F. [Vulnerability assessment of general hospitals of Tehran University of Medical Sciences (Persian)] Journal of Health Promotion Management. 2014; 3(2):65-71.

[23] Asefzadeh S, Safari Varyani S, Gholami S. Disaster risk assessment in educational hospitals of Qazvin based on WHO pattern in 2015. Electronic Physician. 2016; 8(1):1770-75. PMCID: PMC4768927 